\title{
Combination of Antibiotics- Nisin Reduces the Formation of Persister Cell in Listeria monocytogenes
}

\author{
Negar Narimisa, ${ }^{1,2}$ Behrooz Sadeghi Kalani, ${ }^{3,4}$ \\ Rokhsareh Mohammadzadeh, ${ }^{2}$ and Faramarz Masjedian Jazi ${ }^{1,2}$
}

Persister cells are a subpopulation of bacteria with the ability of survival when exposed to lethal doses of antibiotics, and are responsible for antibiotic therapy failure and infection recurrences. In this study, we investigated persister cell formation and the role of nisin in combination with antibiotics in reducing persistence in Listeria monocytogenes. We also examined the expression of toxin-antitoxin (TA) systems in persister cells of $L$. monocytogenes to gain a better understanding of the effect of TA systems on persister cell formation. To induce persistence, L. monocytogenes were exposed to high doses of different antibiotics over a period of $24 \mathrm{hr}$, and the expression levels of TA system was genes were measured $5 \mathrm{hr}$ after the addition of antibiotics by the quantitative reverse transcription-polymerase chain reaction (qRT-PCR) method. To investigate the effect of nisin, $L$. monocytogenes was exposed to a combination of nisin and antibiotics. According to our results, $L$. monocytogenes was highly capable of persister cell formation, and the combination of nisin and antibiotics resulted in reduced persistence. qRT-PCR results showed a significant increase in GNAT/RHH expression among the studied systems. Overall, our results demonstrated the potential of the combination of nisin and antibiotics in reducing persister cell formation, and emphasized the role of the GNAT/RHH system in bacterial persistence.

Keywords: Listeria monocytogenes, nisin, persister cells, TA system, real-time PCR

\section{Introduction}

$\mathbf{P}$ ERSISTERS ARE A subset of the bacterial population that can survive lethal doses of various antimicrobial agents. ${ }^{1}$ Bacterial persistence was at first defined by Joseph Bigger in 1944. According to his observations, a small population of Staphylococcus aureus was not killed by penicillin. According to Bigger, these persister cells temporarily enter the dormant state, thereby disabling penicillin to eradicate the infection. ${ }^{2}$ Persister cells are formed when bacterial populations are exposed to increasing or constant concentrations of antibiotics over a long period. ${ }^{3}$

Persisters can tolerate lethal doses of antibiotics by phenotype alterations without any heritable resistance mechanisms. ${ }^{4}$ Distinct from drug-resistant cells, persister cells do not grow in the presence of antibiotics; however, after the elimination of antibiotic pressure, they can regrow and form a population similar to the parental population they originated from. ${ }^{5}$
Bacterial persistence is widely considered as a significant challenge in infection treatment. Studies have shown that persister cell formation not only leads to antimicrobial therapy failure but also boosts the progression of resistance. ${ }^{6}$ Persister cells have been found in many bacterial species, such as Klebsiella pneumoniae, S. aureus, Pseudomonas aeruginosa, and Mycobacterium tuberculosis, and they play an important role in the recurrence of microbial infections. ${ }^{7,8}$

Listeria monocytogenes is a Gram-positive bacterium leading to severe food-borne infections in immunocompromised humans, elderly, neonates, and pregnant women. ${ }^{9}$ L. monocytogenes is widely distributed and isolated from a variety of foods, including fruits, vegetables, raw dairy, meat, seafood, and ready-to-eat (RTE) foods. ${ }^{10,11}$ L. monocytogenes, as one of the most important food-borne pathogens with high mortality and high hospitalization rates, has become a major concern for food manufacturers as well as the health and regulatory officials. ${ }^{12}$ L. monocytogenes is

\footnotetext{
${ }^{1}$ Microbial Biotechnology Research Center, Iran University of Medical Science, Tehran, Iran.

${ }^{2}$ Department of Microbiology, School of Medicine, Iran University of Medical Sciences, Tehran, Iran.

${ }^{3}$ Department of Medical Microbiology, Faculty of Medicine, Ilam University of Medical Sciences, Ilam, Iran.

${ }^{4}$ Clinical Microbiology Research Center, Ilam University of Medical Sciences, Ilam, Iran.
} 
TABle 1. Listeria monocytogenes Strains Used IN THIS STUDY

\begin{tabular}{lllc}
\hline Strain & \multicolumn{1}{c}{ Description } & Source & Reference \\
\hline LM 1 & $\begin{array}{c}\text { A clinical isolate belonging } \\
\text { to serotype 1/2c, 3c }\end{array}$ & Milk & 36 \\
LM 2 & $\begin{array}{c}\text { A clinical isolate belonging } \\
\text { to serotype 4c } \\
\text { clinical isolate belonging } \\
\text { to serotype1/2c, 3c } \\
\text { LM 3 }\end{array}$ & Stool & 36 \\
LM 4 & $\begin{array}{c}\text { Alinical isolate belonging } \\
\text { to serotype 1/2a, 3a } \\
\text { clinical isolate belonging } \\
\text { to serotype 1/2a, 3a }\end{array}$ & $\begin{array}{c}\text { Trout } \\
\text { Placental } \\
\text { bit }\end{array}$ & 36 \\
\hline
\end{tabular}

sensitive to a wide range of antibiotics. The treatment of choice for listeriosis includes ampicillin alone or in combination with gentamicin. ${ }^{13}$ However, this bacterium is inherently resistant to several antibiotics, including the broadspectrum cephalosporins and fosfomycin. Moreover, the treatment of listeriosis is complicated since not only $L$. monocytogenes is an intracellular pathogen causing the reduced effect of antibiotics, but also most antibiotics have bacteriostatic effects on this bacterium. ${ }^{14,15}$ Another reason for treatment failure could be persister cell formation, which allows bacteria to cope with antibiotic treatment. ${ }^{16}$

There are many mechanisms involved in persister cell formation, and one of the most studied mechanisms is the toxin-antitoxin (TA) systems. ${ }^{17}$ TA systems are small genetic elements widely present on bacterial plasmids and chromosomes. TA systems consist of two genes coding for the stable toxin and its unstable cognate antitoxin. TAs are currently divided into six distinct classes. Among all TA systems, type II and their role in persistence have been extensively studied. ${ }^{18}$ In type II systems, antitoxin is a small unstable protein inhibiting toxin activity through proteinprotein complex formation. In stress conditions, such as antibiotic exposure, antitoxins are degraded by cellular proteases, and the free active toxins interfere with essential cellular processes (replication, cell-wall synthesis, and translation), thereby reducing bacterial growth and inducing dormancy and persistence. ${ }^{17,19}$
Therefore, this study aimed to evaluate persister cell formation after exposure to different antibiotics and to investigate the antipersister potential of nisin (as an antimicrobial peptide) in combination with antibiotics. Moreover, due to the role of TA systems in bacterial persistence, the effects of lethal concentrations of selected antibiotics were analyzed on the expression levels of type II TA systems in L. monocytogenes persister cells.

\section{Materials and Methods \\ Bacterial strains and growth conditions}

In this study, L. monocytogenes ATCC 19115 and 5 clinical strains were used (Table 1). Bacterial strains were stored in Brain Heart Infusion (BHI) broth (Merck, Darmstadt, Germany) containing $20 \%$ glycerol at $-80^{\circ} \mathrm{C}$.

\section{Determination of the minimum inhibitory concentration}

Minimum inhibitory concentrations (MICs) of ampicillin, gentamicin, ciprofloxacin (Sigma Aldrich), and nisin $(2.5 \%$ [wt/wt], balanced $\mathrm{NaCl}$ and denatured milk solids; Sigma Aldrich) against L. monocytogenes were determined by the broth microdilution method using 96 U-shaped well plates. ${ }^{20}$

After adding $100 \mu \mathrm{L}$ of Trypticase Soy Yeast Extract (TSYE) broth (Oxoid, United Kingdom) to each well of a 96-well plate, $100 \mu \mathrm{L}$ of each antibiotic solution was added to the first well and serial dilution was prepared. Finally, $100 \mu \mathrm{L}$ of the overnight culture containing $\sim 10^{5}$ cells was added to each well. Plates were incubated at $37^{\circ} \mathrm{C}$ for $24 \mathrm{hr}$, and the MICs were determined by direct observation. MIC values for the studied antibiotics were determined independently in triplicates.

\section{Persister formation assays}

Persister cell formation in L. monocytogenes was investigated by exposing L. monocytogenes culture in the exponential phase to high concentrations of bactericidal antibiotics. To measure the number of persister cells, a single colony of L. monocytogenes was inoculated into $5 \mathrm{~mL}$ BHI broth (Merck) for $24 \mathrm{hr}$. Fifty microliters of this subculture was resuspended in $50 \mathrm{~mL}$ of BHI broth and bacterial culture was incubated at $37^{\circ} \mathrm{C}$ on a shaker at $200 \mathrm{rpm}$ until the optical density at $600 \mathrm{~nm}$ reached 0.25 . To obtain

Table 2. Primers Used for Quantitative Reverse Transcription-Polymerase Chain Reaction

\begin{tabular}{|c|c|c|c|}
\hline Primer name & Primer sequence & Product size (bp) & Reference \\
\hline \multirow[t]{2}{*}{ GNAT } & F: GGAACCAAGGCTATGCCACT & \multirow[t]{2}{*}{199} & \multirow[t]{2}{*}{35} \\
\hline & R: ACATGGGGCTTTAGTGGGTC & & \\
\hline \multirow[t]{2}{*}{$\mathrm{RHH}$} & F: ACGAAGCAGAGACACCCAAT & \multirow[t]{2}{*}{245} & \multirow[t]{2}{*}{35} \\
\hline & R: TACAACGATTTCGCCATTGA & & \\
\hline \multirow[t]{2}{*}{ COG } & F: CCAAGACGAGGTAACAATCAGA & \multirow[t]{2}{*}{142} & \multirow[t]{2}{*}{35} \\
\hline & R: TTTGTAATGCATCAATTCCTCTACT & & \\
\hline \multirow[t]{2}{*}{ Xre } & F: GTTGATGAACGCTTGGTGAC & \multirow[t]{2}{*}{66} & \multirow[t]{2}{*}{35} \\
\hline & R: CCGCATTTCTTCTTCGGT & & \\
\hline \multirow[t]{2}{*}{ mazF } & F: GGAATACGGCCTGTTCTCAT & \multirow[t]{2}{*}{125} & \multirow[t]{2}{*}{35} \\
\hline & R: CGAGTAGCTTCCACGTGTGT & & \\
\hline \multirow[t]{2}{*}{ mazE } & F: ATGGGGCGGAGTGAAGTTAT & \multirow[t]{2}{*}{160} & \multirow[t]{2}{*}{35} \\
\hline & R: CTTCTGCTTCTGCTTCGACA & & \\
\hline \multirow[t]{2}{*}{ 16S rRNA } & F: ATGGCCTACCAAGGCAACGA & \multirow[t]{2}{*}{83} & \multirow[t]{2}{*}{35} \\
\hline & R: GTAGGAGTCTGGGCCGTGTC & & \\
\hline
\end{tabular}


Table 3. Antibiotic Susceptibility Profiles OF LISTERIA MONOCYTOGENES ATCC 19115

\begin{tabular}{lc}
\hline Antibiotics & $M I C(\mu \mathrm{g} / \mathrm{mL})$ \\
\hline Ampicillin & 0.031 \\
Gentamicin & 1 \\
Ciprofloxacin & 1 \\
Nisin & 8 \\
\hline
\end{tabular}

MIC, minimum inhibitory concentration.

concentration-dependent killing curves, L. monocytogenes culture in the exponential phase was treated with 10-, 40-, 100-, and 200-fold MICs of ampicillin, gentamicin, and ciprofloxacin, and cultures were incubated at $37^{\circ} \mathrm{C}$ ( $200 \mathrm{rpm})$ for $4 \mathrm{hr}$. Time-dependent killing curves were determined by challenging the culture with 100 -fold MICs of the antibiotics for $24 \mathrm{hr}$. At 1, 3, 5, and $24 \mathrm{hr}$ after antibiotic challenge, bacterial cells were washed twice with $0.85 \%$ sterile saline solution to remove the antibiotics and after preparing the serial dilutions of bacterial culture, each dilution was inoculated onto BHI agar for colony counting. Bacterial culture without any treatments was selected as the control.

\section{Persister cell formation after combination treatment with nisin and the studied antibiotics}

To determine the concentration-dependent killing curves in the presence of nisin, L. monocytogenes ATCC 19115 culture in the exponential phase was exposed to 100-fold MICs of ampicillin, gentamicin, and ciprofloxacin in the presence of different concentrations of nisin $(0-400 \mu \mathrm{g} / \mathrm{mL})$ for $4 \mathrm{hr}$. To determine the time-dependent killing curves in the presence of nisin, L. monocytogenes ATCC 19115 culture in the exponential phase was exposed to 100-fold MICs of the studied antibiotics in the presence of $400 \mu \mathrm{g} / \mathrm{mL}$ nisin for $24 \mathrm{hr}$. The bacterial culture at different time intervals was washed and serially diluted in $0.85 \%$ sterile saline solution, and each dilution was inoculated onto BHI agar to determine the colony-forming units.

\section{Relative quantitative reverse transcription-polymerase chain reaction}

Total RNA was extracted from $1 \mathrm{~mL}$ of L. monocytogenes ATCC 19115 in the exponential phase after $5 \mathrm{hr}$ of treatment with 100-fold MICs of ampicillin, gentamicin, ciprofloxacin, and nisin. Total RNA of untreated L. monocytogenes ATCC 19115 was also extracted as the control, using a high pure RNA isolation kit (Roche kit, Germany) according to the manufacturer's protocol. Total RNA was treated with DNase1 (Thermo Scientific) according to the DNase treatment protocol. The RNA quality was assessed using a NanoDrop spectrophotometer (Thermo Scientific) and gel electrophoresis analysis. Total RNA (1 $\mu \mathrm{g})$ was converted to cDNA using the cDNA Synthesis Kit (Thermo Scientific). The sequences of primers used in this study are listed in Table 2. Quantitative reverse transcriptionpolymerase chain reaction (qRT-PCR) was performed on three technical replicates for each sample using a RotorGene thermal cycler (Corbett Life Sciences, Sydney, Australia) with SYBR Green method (Ampliqon Co, Denmark). Total amplification reaction $(20 \mu \mathrm{L})$ consisted of $1 \mu \mathrm{L}$ of cDNA, $10 \mu \mathrm{L}$ SYBR Green master mix, $7 \mu \mathrm{L}$ nuclease-free water, and $1 \mu \mathrm{L}$ of each primer. The thermal program consisted of an initial denaturation at $95^{\circ} \mathrm{C}$ for $12 \mathrm{~min}$, followed by 40 cycles at $95^{\circ} \mathrm{C}$ for $10 \mathrm{sec}, 57^{\circ} \mathrm{C}$ for $20 \mathrm{sec}$, and $72^{\circ} \mathrm{C}$ for $20 \mathrm{sec}$. $16 \mathrm{~S}$ rRNA was used as an internal control to normalize the expression levels of each gene. The relative fold changes in expression levels were measured using the delta-delta $\mathrm{Ct}$ method. ${ }^{21}$
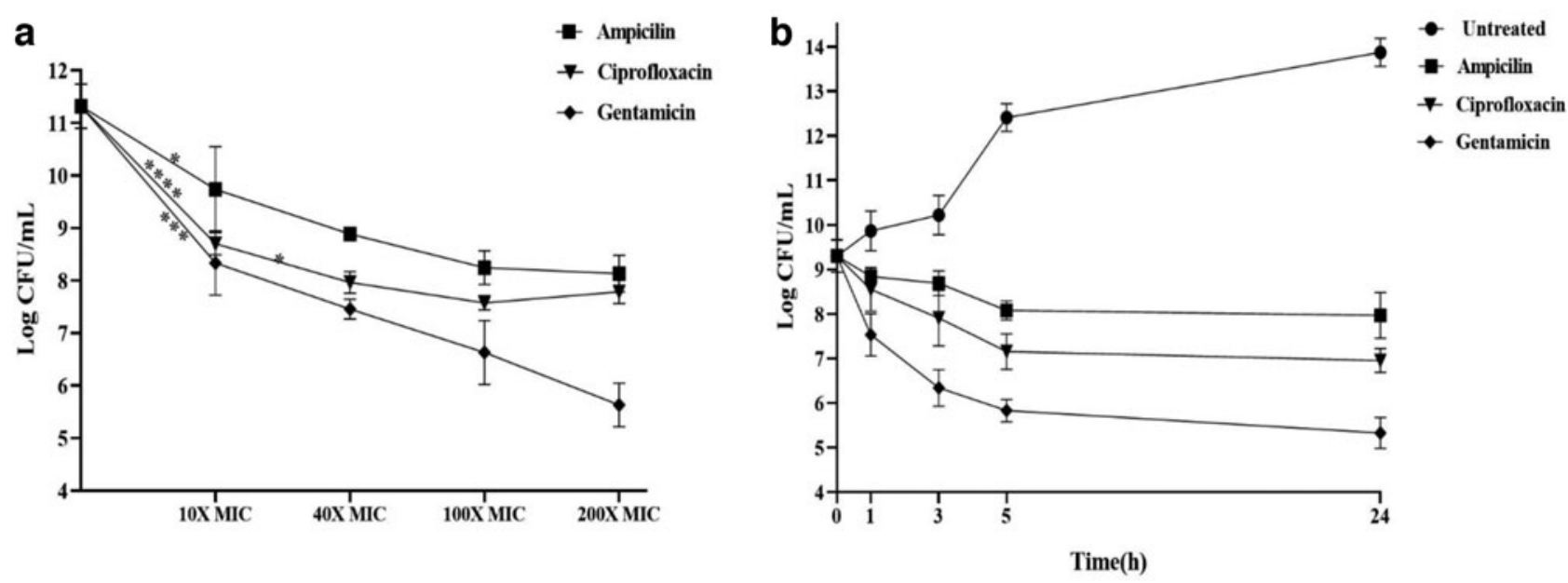

FIG. 1. Persister cell formation in Listeria monocytogenes ATCC 19115. (a) Concentration-dependent killing curves; L. monocytogenes ATCC 19115 in the exponential phase was exposed to increasing concentrations of antibiotics for $4 \mathrm{hr}$. $* p<0.05$; *** $p<0.001$; ****p $\leq 0.0001$ by one-way ANOVA with Tukey's post hoc test for multiple comparisons. (b) Time-dependent killing curves; L. monocytogenes ATCC 19115 in the exponential phase was treated with 100-fold MICs of antibiotics for $24 \mathrm{hr}$. Data represent the mean $( \pm \mathrm{SD})$ of three biological replicates. ANOVA, analysis of variance; MIC, minimum inhibitory concentration; SD, standard deviation. 


\section{Statistical analysis}

Significant differences were assessed using a one-way analysis of variance (ANOVA) followed by Tukey's post hoc test and Dunnett's test for multiple comparisons (as indicated in the figure legends). All data analyses and statistical chart drawings were carried out using GraphPad Prism 8 (GraphPad Software, Inc.).

\section{Results}

\section{Persister cell formation assays}

We first determined the MICs of ampicillin, gentamicin, and ciprofloxacin against L. monocytogenes ATCC 19115 using the broth microdilution method (Table 3). To investigate persister cell formation, we exposed L. monocytogenes ATCC 19115 in the exponential phase to increasing concentrations of antibiotics for $4 \mathrm{hr}$. Concentration-dependent killing curves revealed a maximum rate of persister cell formation against 10-fold MICs of ampicillin $(p<0.05)$. Increased concentrations of ampicillin did not have any effects on bacterial killing. Exposure to ciprofloxacin led to a more effective reduction in the number of viable cells ( $p \leq 0.0001)$ and also increasing the concentration up to 40fold MIC of ciprofloxacin, led to an effective reduction in the number of cells $(p<0.05)$. However, at high concentrations, increasing antibiotic concentrations had no significant effects on the number of cells being killed. In the presence of 10-fold MICs of gentamicin, bacterial viability was effectively reduced) $p \leq 0.001$ ), and an increase in gentamicin concentration had no significant effect on bacterial killing. It was also observed that a smaller fraction of persister cells was observed in the presence of gentamicin than ampicillin and ciprofloxacin (Fig. 1a). Time-dependent killing curves revealed the typical biphasic killing patterns with a sharp decline in the susceptible cells followed by a plateau of the surviving persister subpopulation. We performed time-dependent killing experiments by exposing $L$. monocytogenes ATCC 19115 culture in the exponential phase to the 100-fold MICs of ampicillin, gentamicin, and ciprofloxacin over a $24 \mathrm{hr}$ period. According to the results,
Table 4. Minimum Inhibitory Concentration ( $\mu \mathrm{G} / \mathrm{ML})$ of AMPICILlin AgAinst DiffERENT LISTERIA MONOCYTOGENES STRAINS

\begin{tabular}{lc}
\hline Isolate & $M I C(\mu \mathrm{g} / \mathrm{mL})$ \\
\hline LM 1 & 0.062 \\
LM 2 & 0.125 \\
LM 3 & 0.031 \\
LM 4 & 0.025 \\
LM 5 & 0.125
\end{tabular}

exposure to gentamicin $(100 \times$ MIC) induced the lowest level of persister cell formation $(5.3 \log \mathrm{CFU} / \mathrm{mL})$. The rate of persister cell formation in the presence of ciprofloxacin $(100 \times \mathrm{MIC})$ was $6.9 \log \mathrm{CFU} / \mathrm{mL}$, and the maximum persister cell formation (7.9 $\log \mathrm{CFU} / \mathrm{mL}$ ) was observed in the presence of ampicillin $(100 \times \mathrm{MIC})($ Fig. 1b). These results indicate that L. monocytogenes ATCC 19115 can tolerate high concentrations of bactericidal antibiotics by forming persister cells. Moreover, according to these results, the rate of persister cell formation may differ depending on the type of antibiotics.

We selected five clinical strains of L. monocytogenes to analyze persister cell formation in clinical strains. First, we determined the MICs of ampicillin against these strains. The MIC values of ampicillin for each strain are shown in Table 4. Strains were exposed to the 100-fold MICs of ampicillin for $24 \mathrm{hr}$. The typical biphasic killing curves were formed by an initial rapid killing followed by a plateau of surviving drug-tolerant cells for all the studied strains over a $24 \mathrm{hr}$ period (Fig. 2). According to these results, clinical strains of L. monocytogenes are capable of producing high levels of persister cells, demonstrating the importance of persister cell formation in tolerating high doses of antibiotics.

\section{Reduced persister cell formation by combination treatment of nisin and the studied antibiotics}

To investigate the ability of bacterial killing of the combination of antibiotics and nisin, we first treated
FIG. 2. Persister cell formation in different Listeria monocytogenes clinical strains. Clinical strains in the exponential phase were treated with 100-fold MICs of antibiotics for $24 \mathrm{hr}$. Data represent the mean $( \pm \mathrm{SD})$ of three biological replicates.

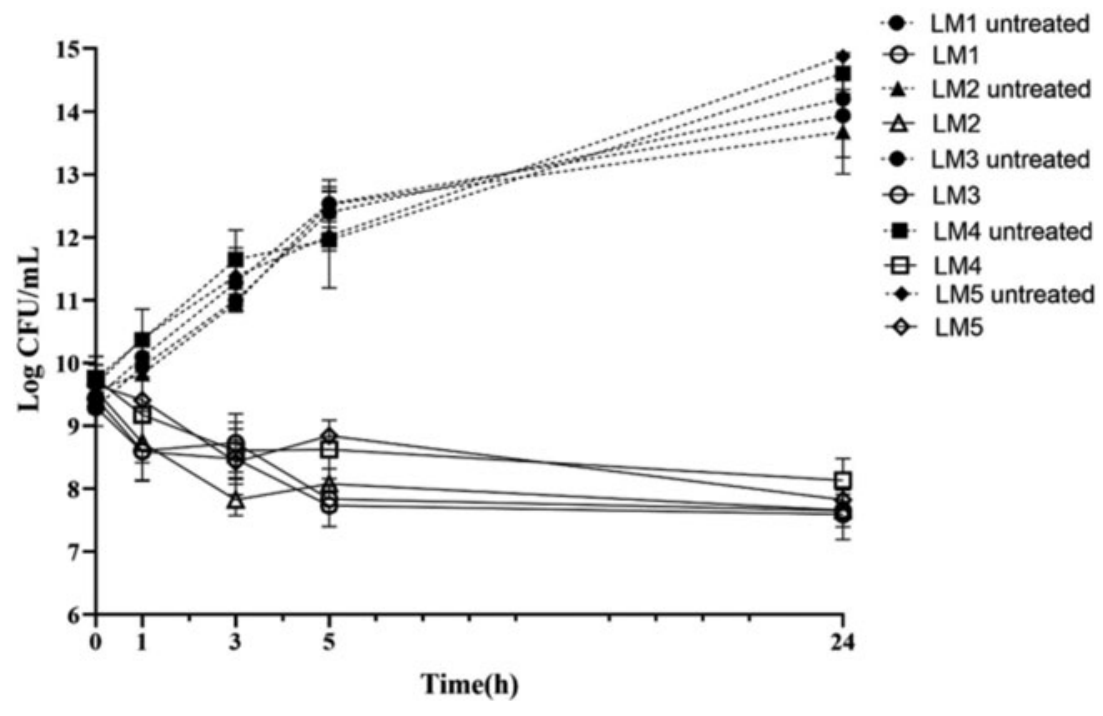


L. monocytogenes ATCC 19115 in the exponential phase with 100-fold MICs of ampicillin, gentamicin, and ciprofloxacin and different concentrations of nisin for $4 \mathrm{hr}$. Our results showed that in the presence of ciprofloxacin and gentamicin increased nisin concentrations could effectively reduce persister cell formation within $4 \mathrm{hr}$. The presence of $400 \mu \mathrm{g} / \mathrm{mL}$ of nisin with 100-fold MICs of ciprofloxacin resulted in a $1.6 \log$-fold reduction) $p \leq 0.001$ ) and also a $1.93 \mathrm{log}$-fold reduction against 100 -fold MIC of gentamicin) $p \leq 0.0001)$ in persister cell formation. The presence of $400 \mu \mathrm{g} / \mathrm{mL}$ of nisin with 100 -fold MIC of ampicillin with a reduction of 1 log-fold showed that increasing nisin concentration over $4 \mathrm{hr}$ had no significant effect on persister cell formation in the presence of ampicillin (Fig. 3a). The timedependent assay showed that the combination of $400 \mu \mathrm{g} / \mathrm{mL}$ nisin with 100 -fold MIC of gentamicin) $p \leq 0.01$ ), ciprofloxacin) $p \leq 0.05$ ), and ampicillin) $p \leq 0.001$ ) was significantly effective in decreasing persister cell formation of L. monocytogenes over a $24 \mathrm{hr}$ period (Fig. 3b). Considering these observations, this study showed that the combination of nisin with antibiotics might be useful for the eradication of persister cells.
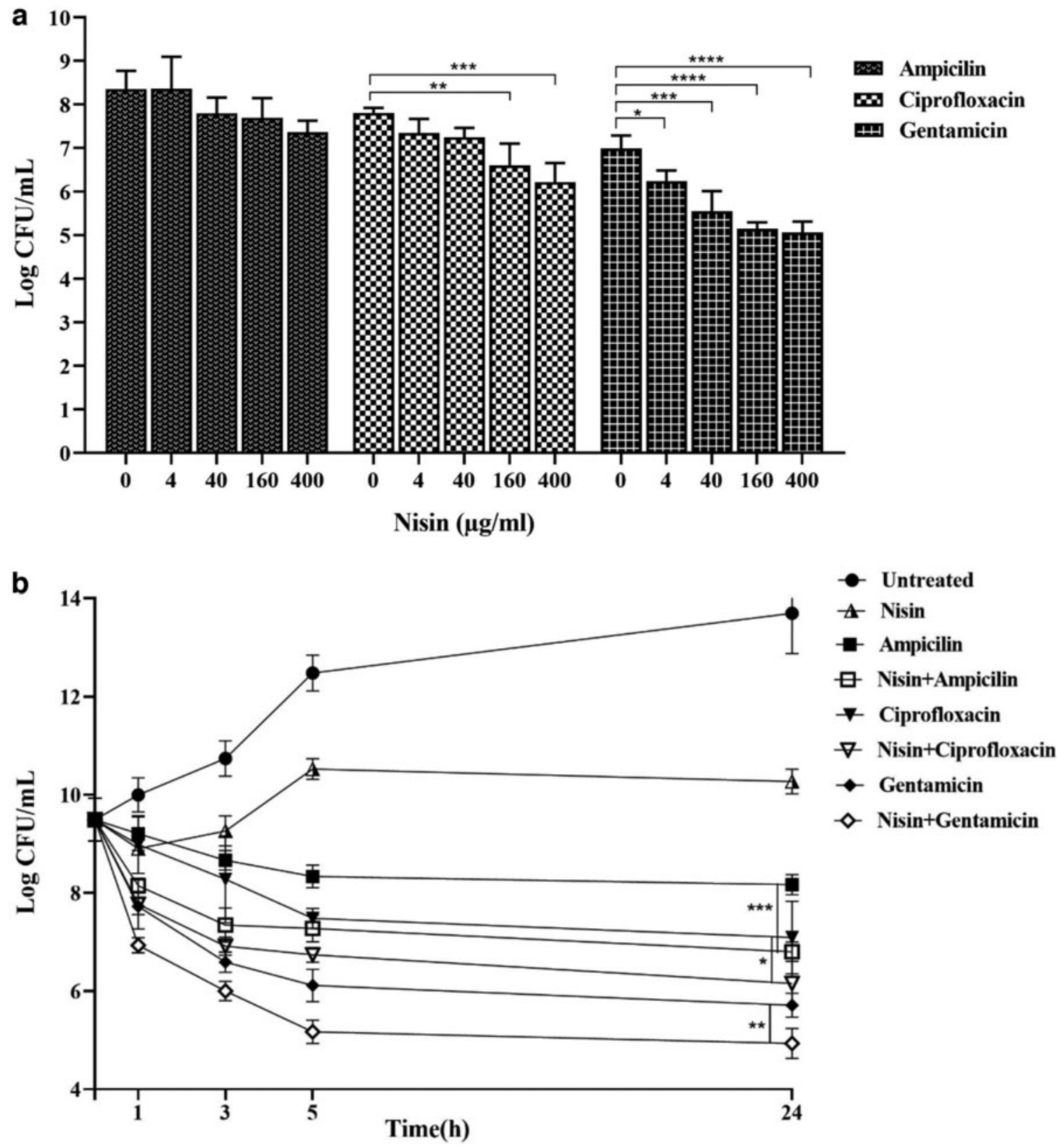

FIG. 3. Effect of nisin on persister cell formation in Listeria monocytogenes ATCC 19115. (a) L. monocytogenes ATCC 19115 in the exponential phase was treated with different concentrations of nisin in combination with 100-fold MICs of antibiotics for $4 \mathrm{hr}$. $* p<0.05$; $* * p<0.01$; $* * * p<0.001$; **** $p \leq 0.0001$ by one-way ANOVA with Dunnett's multiple comparisons test. (b) Effect of nisin $(400 \mu \mathrm{g} / \mathrm{mL})$ on persister cell formation in L. monocytogenes ATCC 19115 in the exponential phase against 100-fold MICs of antibiotics. Data represent the mean $( \pm \mathrm{SD})$ of three biological replicates. $* p<0.05 ; * * p<0.01 ; * * * p<0.001$ by one-way ANOVA with Tukey's post hoc test for multiple comparisons. 


\section{Relative $q R T-P C R$}

To evaluate the expression of TA system genes, after $5 \mathrm{hr}$ of exposure of L. monocytogenes ATCC 19115 culture in the exponential phase to a lethal dose $(100 \times$ MIC) of ampicillin, ciprofloxacin, gentamicin, and nisin, RNA of this bacterial culture was extracted, and the relative expression levels of TA system genes were determined. RNA of the untreated culture was used as the control.

The results of the qRT-PCR assay revealed that the expression of the GNAT/RHH system significantly increased in the presence of lethal doses of all three antibiotics. The expression of the $\mathrm{COG} / \mathrm{Xre}$ system increased in the presence of ampicillin and ciprofloxacin. The presence of gentamicin also led to a slight increase in the expression of this system. According to our results, the expression of GNAT/RHH system genes was the lowest in the presence of nisin, which may indicate that nisin is less effective in the activation of this system. The expression of the MazEF system was downregulated upon exposure to all three antibiotics relative to the control sample (Fig. 4). According to these results, the GNAT/RHH system can have a major impact on bacterial persistence.
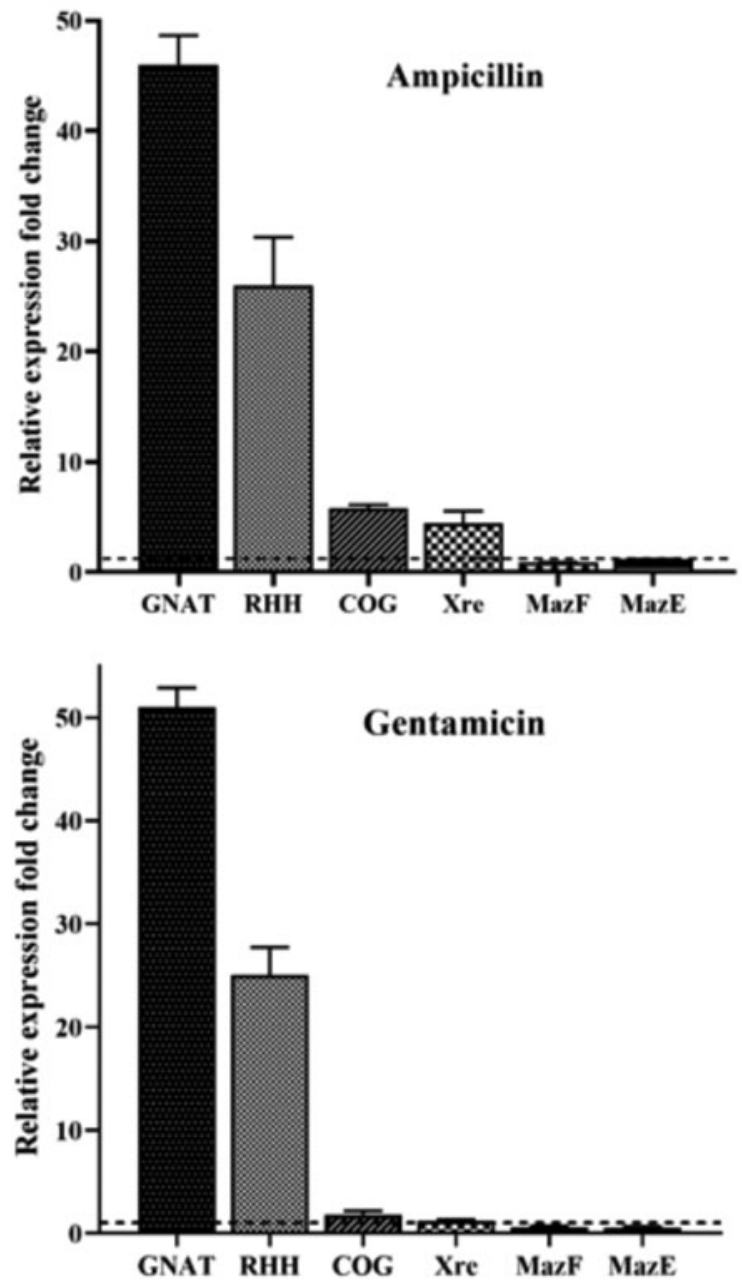

\section{Discussion}

Currently, persister cell formation in bacteria is identified as one of the main causes of antibiotic therapy failure and infection recurrences. ${ }^{22}$ Persister cell formation occurs not only in bacteria but also in eukaryotes, including yeast and even human cancer cells, leading to antibiotic therapy failure. $^{3,23}$ Understanding the mechanisms of persister cell formation is crucial for enhancing our ability to fight the crisis of chronic bacterial infections and antibiotic therapy failure.

Variations in persister cell formation against different antibiotics have been reported in some bacteria, including Acinetobacter baumannii and $K$. pneumoniae. ${ }^{24,25}$ The study by Hofsteenge et al. demonstrated that no direct and positive correlation was found in persister fractions of Escherichia coli isolates when exposed to ampicillin, ciprofloxacin, and nalidixic acid. According to these results in a bacterial population, different types of persister cells are present, some being tolerant to a single antibiotic, while others being tolerant to several antibiotics, and this difference is extremely dependent on the type of antibiotic and its mechanism of action. ${ }^{26}$
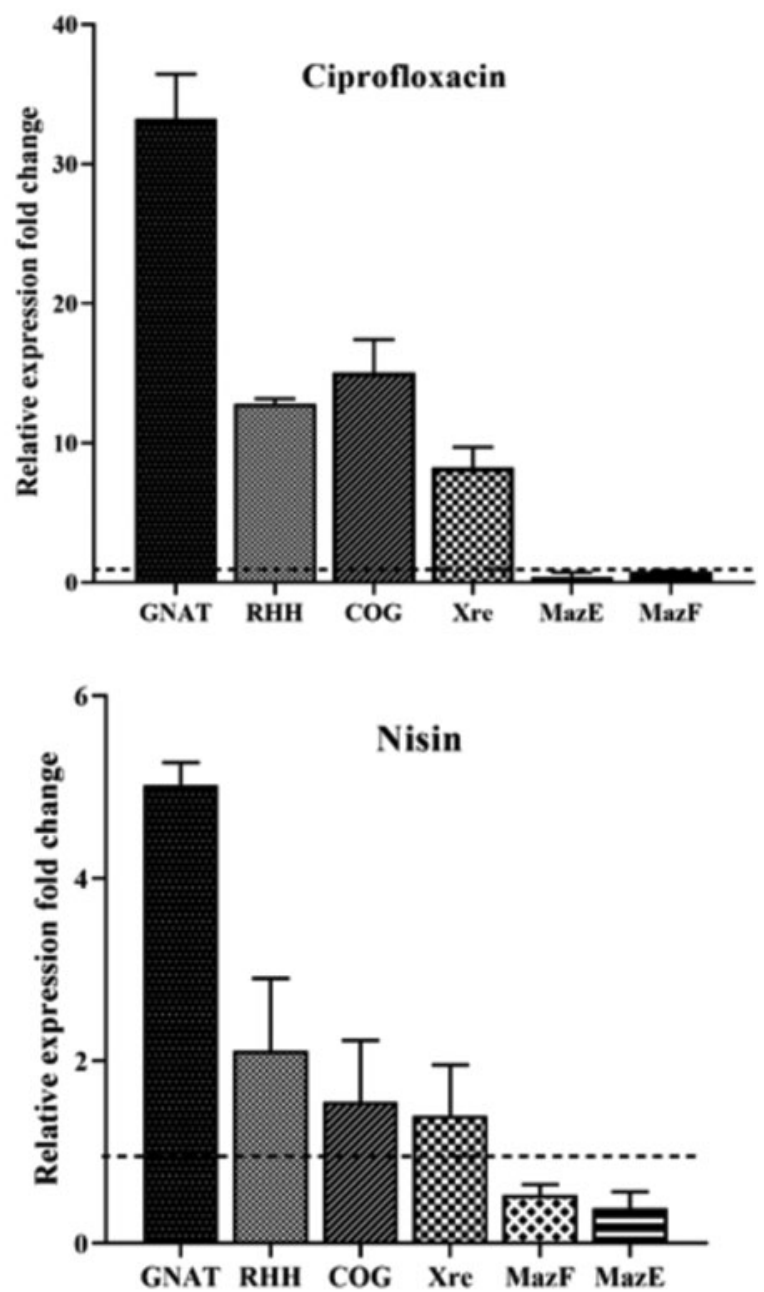

FIG. 4. Relative expression of TA system genes in Listeria monocytogenes ATCC 19115 in the exponential phase exposed to 100-fold MICs of ampicillin, ciprofloxacin, gentamicin, and nisin. Bars represent the mean \pm SD of three biological replicates. TA, toxin-antitoxin. 
In our study, we investigated the presence of persister cells in the L. monocytogenes ATCC 19115 population after exposure to various bactericidal antibiotics commonly used for the clinical treatment of $L$. monocytogenes infections. This study showed that $L$. monocytogenes produced different levels of persister cells against different bactericidal antibiotics, including ampicillin, ciprofloxacin, and gentamicin from the highest level to the lowest level, respectively. This diversity in persister cell formation can be due to variations in the function of different antibiotics.

We also investigated persister cell formation in L. monocytogenes culture upon exposure to various high concentrations of bactericidal antibiotics with nisin. Nisin is a microbial-derived antimicrobial compound used as a preservative in the food industry. ${ }^{27}$ Nisin can control pathogens by inducing pore formation in cell membranes and hindering cell-wall synthesis. According to several reports, combined therapies with nisin improve the antimicrobial activity, reduce the required concentration of antibiotics for effective therapies, and to some extent reduce the potential toxic or adverse effects of antibiotics. ${ }^{28}$ In P. aeruginosa infections, the combination of nisin and polymyxin showed a synergistic antimicrobial activity, reducing the required concentrations of polymyxin to inhibit biofilm formation. In fact, this synergistic effect is because nisin and polymixin have different mechanisms of action and target sites. ${ }^{29}$ The study by Rishi et al. showed that administration of nisin (an antimicrobial peptide) with ampicillin would significantly eliminate Salmonella persister cells. ${ }^{30}$ In our study, although the combination of nisin with antibiotics was not able to kill all persister cells, it significantly reduced the survival of persister cells, indicating the potential of nisin as an option for eliminating persister cells.

One of the most investigated mechanisms regarding persister cell formation is the TA systems. Chromosomal TA systems are associated with bacterial dormancy and antibiotic tolerance. ${ }^{17}$ One of the type II TA system toxins is the GCN5related $\mathrm{N}$-acetyltransferase (GNAT). GNAT is a superfamily of proteins with the ability to transfer an acetyl group from acetyl-coA to a wide range of substrates. ${ }^{31}$ In Salmonella, GNAT acetylates aminoacyl-tRNA molecules, inhibits translation, and promotes persister cell formation. ${ }^{32}$ In this study, we investigated the expression of three types of type II TA system genes in the persister cells of L. monocytogenes. Our results showed a significant increase in the expression of GNAT/RHH system in the presence of all antibiotics, including ampicillin, gentamicin, ciprofloxacin, and nisin, indicating the importance of these systems in bacterial persistence. MazF is a type II TA system whose cellular role in inducing persistence is controversial. In E. coli, MazF toxin with its RNase properties cleaves cellular RNAs leading to growth arrest and increases persistence. ${ }^{33}$ The study by Curtis $e t$ al. showed that the $\Delta$ mazEF mutant did not affect persister formation in L. monocytogenes upon exposure to various lethal doses of antibiotics; however, $\Delta$ mazEF mutant had different effects on bacterial survival when exposed to subinhibitory concentrations of antibiotics. ${ }^{34}$ In this study, the expression of MazEF system genes decreased in the presence of lethal concentrations of the studied antibiotics, suggesting that in L. monocytogenes this system is not involved in persister cell formation and reportedly, it may be involved in responding to other stresses, such as heat and the subinhibitory concentrations of antibiotics. ${ }^{34,35}$

\section{Conclusion}

This study shows that L. monocytogenes is capable of forming persister cells with the ability to tolerate high concentrations of antibiotics. Moreover, our study confirms the effectiveness of the combination of nisin and antibiotics in reducing bacterial persistence. The remarkable increase in the expression of GNAT could indicate the role of this system in persister cell formation, which necessitates further studies for better understanding of the mechanisms of persister cell formation and developing novel approaches to eradicate these cells.

\section{Acknowledgments}

This study was financially supported by Iran University of Medical Sciences (Tehran, Iran), for which we are very grateful.

\section{Disclosure Statement}

No competing financial interests exist.

\section{Funding Information}

This study was supported by a research grant (No. 96-0230-30496) in Iran University of Medical Sciences (Tehran, Iran).

\section{References}

1. Wood, T.K., S.J. Knabel, and B. Kwan. 2013. Bacterial persister cell formation and dormancy. Appl. Environ. Microbiol. 79:7116-7121.

2. Bigger, J. 1944. Treatment of Staphyloeoeeal infections with penicillin by intermittent sterilisation. Lancet 244 : 497-500.

3. Van den Bergh, B., M. Fauvart, and J. Michiels. 2017. Formation, physiology, ecology, evolution and clinical importance of bacterial persisters. FEMS Microbiol. Rev. 41:219-251.

4. Lewis, K. 2010. Persister cells. Annu. Rev. Microbiol. 64: 357-372.

5. Lewis, K. 2005. Persister cells and the riddle of biofilm survival. Biochemistry 70:267-274.

6. Levin-Reisman, I., I. Ronin, O. Gefen, I. Braniss, N. Shoresh, and N. Balaban, 2017. Antibiotic tolerance facilitates the evolution of resistance. Science 355:826830 .

7. Fauvart, M., V.N. De Groote, and J. Michiels. 2011. Role of persister cells in chronic infections: clinical relevance and perspectives on anti-persister therapies. J. Med. Microbiol. 60:699-709.

8. Ren, H., X. He, X. Zou, G. Wang, S. Li, and Y. Wu. 2015. Gradual increase in antibiotic concentration affects persistence of Klebsiella pneumoniae. J. Antimicrob. Chemother. 70:3267-3272.

9. Kalani, B.S., M. Najafi, R. Mohammadzadeh, S. Razavi, E. Ohadi, and G. Irajian. 2019. Targeting Listeria monocytogenes consensus sequence of internalin genes using an antisense molecule. Microb. Pathog. 136:103689.

10. Brigitte, C., and C. Olivier. 2011. Persistence of Listeria monocytogenes in food industry equipment and premises. Int. J. Food Microbiol. 145:1-8.

11. Mohammadzadeh, R., A. Azadegan, and B.S. Kalani. 2019. Listeriolysin S may inhibit the anti-listerial properties of Lactobacillus plantarum. Microb. Pathog. 137:103744. 
12. Donnelly, C. 2001. Listeria monocytogenes: a continuing challenge. Nutr. Rev. 59:183-194.

13. Hof, H., T. Nichterlein, and M. Kretschmar. 1997. Management of listeriosis. Clin. Microbiol. Rev. 10:345-357.

14. Espaze, E., and A. Reynaud. 1988. Antibiotic susceptibilities of Listeria: in vitro studies. Infection 16:S160-S164.

15. Knudsen, G.M., Y. Ng, and L. Gram. 2013. Survival of bactericidal antibiotic treatment by a persister subpopulation of Listeria monocytogenes. Appl. Environ. Microbiol. 79:7390-7397.

16. Cossart, P., and C. Kocks. 1994. The actin-based motility of the facultative intracellular pathogen Listeria monocytogenes. Mol. Microbiol. 13:395-402.

17. Page, R., and W. Peti. 2016. Toxin-antitoxin systems in bacterial growth arrest and persistence. Nat. Chem. Biol. 12:208.

18. Negar, N., A. Fatemeh, K.B. Sadeghi, M. Rokhsareh, and J.F. Masjedian. 2019. Effects of sub-inhibitory concentrations of antibiotics and oxidative stress on the expression of type II TA system genes in Klebsiella pneumoniae. J. Glob. Antimicrob. Resist. [Epub ahead of print]; DOI: 10.1016/ j.jgar.2019.09.005.

19. Yang, Q.E., and T.R. Walsh. 2017. Toxin-antitoxin systems and their role in disseminating and maintaining antimicrobial resistance. FEMS Microbiol. Rev. 41:343-353.

20. Magalhães, L., and M. Nitschke. 2013. Antimicrobial activity of rhamnolipids against Listeria monocytogenes and their synergistic interaction with nisin. Food Control 29: 138-142.

21. Livak, K.J., and T. Schmittgen. 2001. Analysis of relative gene expression data using real-time quantitative PCR and the 2- $\Delta \Delta$ CT method. Methods 25:402-408.

22. Lewis, K. 2010. Persister cells and the paradox of chronic infections. Annu. Rev. Microbiol. 5:429-437.

23. Van Acker, H., P. Van Dijck, and T. Coenye. 2014. Molecular mechanisms of antimicrobial tolerance and resistance in bacterial and fungal biofilms. Trends Microbiol. 22:326-333.

24. Kaur, A., P. Sharma, and N. Capalash. 2018. Curcumin alleviates persistence of Acinetobacter baumannii against colistin. Sci. Rep. 8:11029.

25. Li, Y., L. Zhang, Y. Zhou, Z. Zhang, and X. Zhang. 2018. Survival of bactericidal antibiotic treatment by tolerant persister cells of Klebsiella pneumoniae. J. Med. Microbiol. 67:273-281.

26. Hofsteenge, N., E. van Nimwegen, and O.K. Silander. 2013. Quantitative analysis of persister fractions suggests different mechanisms of formation among environmental isolates of E. coli. BMC Microbiol. 13:25.
27. Guerra, N.P., C.L. Macias, A.T. Agrasar, and L. Castro. 2005. Development of a bioactive packaging cellophane using Nisaplin ${ }^{\circledR}$ as biopreservative agent. Lett. Appl. Microbiol. 40:106-110.

28. Mathur, H., D. Field, M.C. Rea, P.D. Cotter, C. Hill, and R.P. Ross. 2017. Bacteriocin-antimicrobial synergy: a medical and food perspective. Front. Microbiol. 8:1205.

29. Field, D., N. Seisling, P.D. Cotter, R.P. Ross, and C. Hill. 2016. Synergistic nisin-polymyxin combinations for the control of Pseudomonas biofilm formation. Front. Microbiol. 7:1713.

30. Rishi, P., N.R. Bhagat, R. Thakur, and P. Pathania. 2018. Tackling Salmonella persister cells by antibiotic-nisin combination via mannitol. Indian J. Microbiol. 58:239-243.

31. Yeo, C.C. 2018. GNAT toxins of bacterial toxin-antitoxin systems: acetylation of charged tRNAs to inhibit translation. Mol. Microbiol. 108:331-335.

32. Cheverton, A.M., B. Gollan, M. Przydacz, C.T. Wong, A. Mylona, S.A. Hare, and S. Helaine. 2016. A Salmonella toxin promotes persister formation through acetylation of tRNA. Mol. Cell. 63:86-96.

33. Tripathi, A., P.C. Dewan, S.A. Siddique, and R. Varadarajan. 2014. MazF-induced growth inhibition and persister generation in Escherichia coli. J. Biol. Chem. 289:41914205.

34. Curtis, T., I. Takeuchi, L. Gram, and G. Knudsen. 2017. The influence of the toxin/antitoxin mazEF on growth and survival of Listeria monocytogenes under stress. Toxins 9:31.

35. Kalani, B.S., G. Irajian, L. Lotfollahi, E. Abdollahzadeh, and S. Razavi. 2018. Putative type II toxin-antitoxin systems in Listeria monocytogenes isolated from clinical, food, and animal samples in Iran. Microb. Pathog. 122: $19-24$.

36. Zamani, M., F.M. Jazi, B.S. Kalani, and N. Khodaei. 2019. Prevalence of Premature Stop Codons (PMSCs) in Listeria monocytogenes isolated from clinical and food samples in Iran. Gene. Rep. 17:100451.

Address correspondence to:

Faramarz Masjedian, PhD

Department of Medical Microbiology School of Medicine

Iran University of Medical Sciences Shahid Hemmat Highway Tehran 144961-4535 Iran

E-mail: masjedian.f@iums.ac.ir 